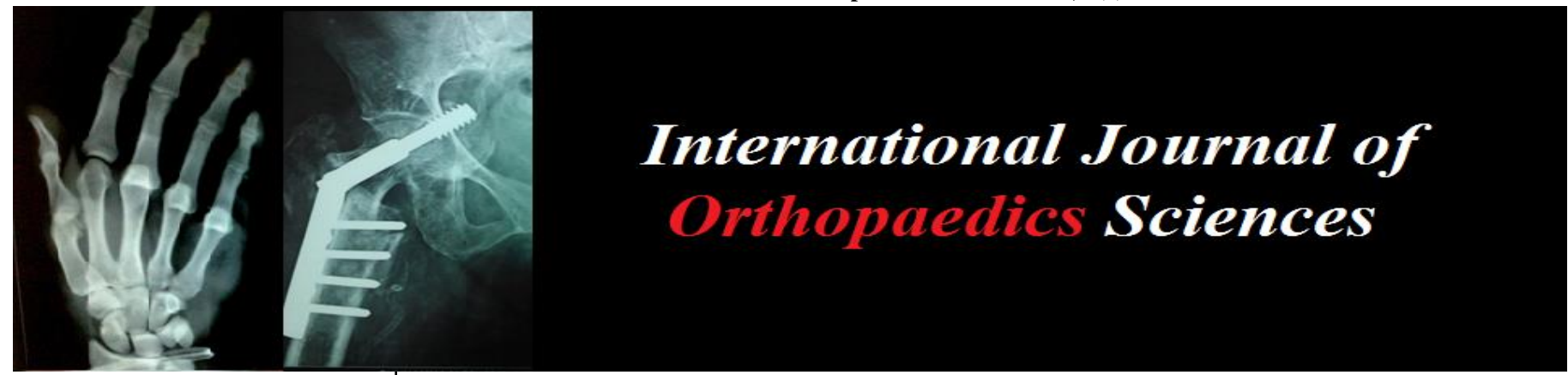

ISSN: $2395-1958$

IJOS 2018; 4(3): 47-55

(C) 2018 IJOS

www.orthopaper.com

Received: 11-05-2018

Accepted: 12-06-2018

Dr. Anil Kumar Mishra

Department of Orthopedics, Armed Forces Medical College,

Pune, Maharashtra, India

Dr. Girish S

Department of Orthopedics, Armed Forces Medical College, Pune, Maharashtra, India

Correspondence

Dr. Girish S

Department of Orthopedics, Armed Forces Medical College, Pune, Maharashtra, India

\section{A prospective study of functional outcome of ACL reconstruction with quadrupled semitendinosus tendon autograft using Endobutton and bioabsorbable interference screw}

\author{
Dr. Anil Kumar Mishra and Dr. Girish S
}

DOI: $\underline{\text { https://doi.org/10.22271/ortho.2018.v4.i3b.11 }}$

\section{Abstract}

Background: The Anterior Cruciate ligament (ACL) is the primary stabilizer of the knee and prevents the knee against anterior translation. It is also important in counteracting rotational and valgus stress. Anterior Cruciate Ligament (ACL) reconstruction is an established and widely practiced surgical procedure with proven efficacy and a low morbidity profile. The arthroscopically aided approach has the advantages of smaller skin and capsular incisions, improved viewing of the intercondylar notch for placement of the tunnel and attachment sites, less postoperative pain, fewer adhesions, earlier motion, and easier rehabilitation. The advantages with semitendinosus grafts are availability of a greater sectional area and unaffected integrity of the extensor mechanism. Moreover tensile strength of quadrupled hamstring is found to be even better than a normal ACL. The rate of complications like extension loss, anterior knee pain and surgical site infections was also lower than other procedures like in BPTB grafts. Cortical suspension devices have the necessary biomechanical properties with regard to ultimate failure strength, displacement, and stiffness for initial fixation of soft tissue in the femoral tunnel for ACL reconstruction. The aperture fixation methods like the interference screws allows for early firm fixation and heal with tight bone-tendon interface.

Objectives: To evaluate functional outcome of arthroscopy assisted ACL reconstruction with quadrupled semitendinosus tendon graft using Endobutton and Bioabsorbable interference screw.

Methodology: The study results included 120 patients (serving soldiers) admitted with clinical and radiological deficiency of ACL during march 2015 to march 2018, in Department of Orthopaedics, Armed Forces Medical College, Pune. Study aims to assess the functional outcome of arthroscopy assisted anterior cruciate ligament reconstruction with quadrupled semitendinosus tendon graft using Endobutton and Bioabsorbable interference screw in terms of range of motion, postoperative knee stability, graft site morbidity, subjective knee functions and prospective assessment of functional outcome using Tegner Lysholm knee scoring system.

Results: All the patients included in the study were serving male soldiers. The analysis of the studied cases revealed that the most common affected age group was between 18 to 24 years $(n=41,34.2 \%)$ and the mean age was $30.53 \pm 7.24$ years. Majority of patients $(n=76,64.4 \%)$ were operated more than 6 months after injury. Sports/BPET (Battle Physical Efficiency Test) injuries was the most common mode of injury ( $\mathrm{n}=79,65.8 \%)$. The mean size of graft used was $8.2 \pm 0.39$.Post-operatively at 02 year follow up, 08 case $(6.7 \%)$ had a positive Pivot shift test with a "Glide" (Grade-I), whereas remaining 112 cases $(93.3 \%)$ had a negative Pivot shift test. None of the patients had grade III laxity. The mean postoperative rolimeter measurement at 02 year follow up was $3.86 \pm 1.06 \mathrm{SD}$. The mean postoperative Lysholm score improved from pre-operative score of $66 \pm 5.2$ to $88.70 \pm 5.54$. The overall satisfactory result (excellent + good) at 02 year follow up was $95 \%$. Out of this, 111(92.5) patients had returned to preinjury level of activity i.e., military training activity. In this study, 9 patients had Superficial Infection, 6 patients had Joint effusion in immediate post operative period, 9 patients had knee pain, 7 patients had sense of giving away during exertional activities and 19 patients had Thigh muscle wasting $(2-3 \mathrm{~cm})$

Conclusion: ACL injuries are common in younger age group individuals. Sports related injury and RTA are the most common modes of injury. Arthroscopy assisted ACL reconstruction using Semitendinosus tendon autograft provides a stable knee, reduces postoperative morbidity and enables early rehabilitation. Graft fixation using endobutton and and Bioabsorbable interference screw will help the graft to facilitate graft tunnel healing and also maintain its strength until there is a good graft to bone healing occurs. It allows the patients to return to preinjury level of activity, allows the patient to squat, sit crossed leg and climb stairs without difficulty. Degeneration of the articular surfaces rarely occurs and the re-operation rates are minimal.

Keywords: ACL, semitendinosus tendon graft, Endobutton, Bioabsorbable interference screw

\section{Introduction}

The Anterior Cruciate ligament (ACL) is the primary stabilizer of the knee and prevents the knee against anterior translation. 
It is also important in counteracting rotational and valgus stress ${ }^{[1]}$. The anterior cruciate ligament (ACL) is one of the most frequently injured ligaments in the human body. Estimated incidences of 0.24 to 0.34 ACL injuries per 1000 population per year have been reported. The anterior cruciate ligament (ACL) rupture is the most common serious injury of the knee ${ }^{[2]}$.

Because of its high prevalence of injury, the ACL continues to be intensively studied, and outcomes of ACL surgery receive considerable attention. Anterior Cruciate Ligament (ACL) reconstruction is an established and widely practiced surgical procedure with proven efficacy and a low morbidity profile [3]. Anterior cruciate ligament (ACL) function is closely associated with optimal activation of the muscles surrounding the knee joint. Rupture of the ACL leads to alterations in muscle recruitment patterns. It has been demonstrated that reconstruction of the ACL re-establishes muscle activation levels ${ }^{[4]}$. This has included research on technical factors such as tunnel position, graft choices, and fixation methods, as well as postoperative rehabilitation protocols. As a result, ACL reconstruction techniques have improved significantly over the last several decades ${ }^{[5]}$.

Arthroscopic techniques have been advanced and refined to assist in the reconstruction of the anterior and posterior cruciate ligaments. The arthroscopically aided approach has the advantages of smaller skin and capsular incisions, improved viewing of the intercondylar notch for placement of the tunnel and attachment sites, less postoperative pain, fewer adhesions, earlier motion, and easier rehabilitation [6]. Arthroscopically assisted ACL reconstruction using a hamstring or patella-bone- tendon-bone auto-graft is the standard surgical treatment particularly for those who are unable to perform jumping and cutting manoeuvres in sports because of resulting knee instability ${ }^{[7]}$.

Trans-portal approach for femoral tunnel preparation and placement of graft is widely used by arthroscopic surgeon with certain advantage over trans-tibial approach. Adoption of this approach has been shown improve femoral tunnel placement in terms of visualization, long horizontal tunnel placement and fewer chances for posterior blow out. Last but not the least it has obviate the need for notch plasty which is a regular procedure in transtibial approach ${ }^{[8]}$.

ACL reconstruction with soft parts (semitendinosus - gracilis muscles), a method more frequently used nowadays ${ }^{[9]}$. The semitendinosus tendon autograft is becoming the graft of choice in anterior cruciate ligament (ACL) reconstruction. The graft with four strands of STG tightened identically has a theoretical advantage over bone patellar tendon bone graft as its mechanical resistance is superior. There are no iatrogenic complications to extensor apparatus and no anterior knee pain. The advantages with semitendinosus grafts are availability of a greater sectional area and unaffected integrity of the extensor mechanism. Moreover tensile strength of quadrupled hamstring is found to be even better than a normal ACL ${ }^{[10]}$. Many studies have found that the rate of return to pre-surgical levels of activity was more than $60 \%$ after this procedure and the rate of complications like extension loss, anterior knee pain and surgical site infections was also lower than other procedures like in BPTB grafts ${ }^{[11]}$

Various fixation methods have been described for ACL reconstruction. They can be classified into aperture fixation and suspensory methods. The suspensory methods can be subclassified into cortical, cancellous and cortio-cancellous suspension methods ${ }^{[12]}$. Most commonly used devices for femoral fixation are interference screws, transfix screws and cortical suspension devices. Cortical suspension devices have been widely used in ACL reconstruction for femoral side graft fixation. Various studies have shown that cortical suspension devices have the necessary biomechanical properties with regard to ultimate failure strength, displacement, and stiffness for initial fixation of soft tissue in the femoral tunnel for ACL reconstruction $[13,14,15]$. Devices for tibial fixation can be divided according to the location of fixation: intratunnel fixation and extratunnel fixation. Intratunnel fixation methods primarily rely on interference screw (metallic or bioabsorbable) or a cross pin system ${ }^{[16]}$. The aperture fixation methods like the interference screws allows for early firm fixation and heal with tight bone-tendon interface ${ }^{[17,18]}$.

The objective of this study was to evaluate functional outcome of arthroscopy assisted ACL reconstruction with quadrupled semitendinosus tendon graft using Endobutton and Bioabsorbable interference screw.

\section{Materials and methods Source of Data}

The study was conducted on 137 patients (serving soldiers) admitted with clinical and radiological deficiency of ACL during march 2015 to march 2018, in Department of Orthopaedics, Armed Forces Medical College, Pune. 06 patients during follow up sustained reinjury, requiring revision ACL reconstruction with ipsilateral BPTB autograft and 11 patients were lost to follow up. These 17 patients were excluded from the study. The study results included 120 patients (serving soldiers) with minimum of 2 years follow up.

\section{Inclusion Criteria}

1. Age: 18 to 45 years fighting serving soldiers

2. Isolated ACL injuries/ ACL injuries with grade 1,2 meniscal injuries

\section{Exclusion Criteria}

1. ACL injuries with avulsion injuries or associated intraarticular condylar fractures.

2. Multiligamentous injuries

3. Meniscal injuries requiring partial/total meniscectomy/meniscal repair (following initial diagnostic arthroscopy)

4. Pre-existing:- congenital/developmental/collagen diseases.

5. Infected knee joint.

6. Previous surgery in ipsilateral limb.

\section{Clinical Assessment}

On admission of the patient, a careful history was elicited from the patient to reveal the mechanism of injury and the severity of the trauma. The patients were then evaluated clinically for laxity by Lachman, Ant drawer by rollimeter, pivot shift test, and assessed by Lysholm and Gillquist knee scoring system.

\section{Radiographic Assessment}

Standard guidelines were followed to get radiographs. Anteroposterior and lateral radiographs of the affected knee. All the patients were also evaluated by MRI of knee preoperatively.

\section{Pre Operative Evaluation}

All the patients were explained about the aims of the study, the methods involved and an informed written consent was obtained before being included in the study. All patients with 
clinical and radiological deficiency of ACL were initially evaluated with diagnostic arthroscopy of knee. Patients meeting the inclusion and exclusion criteria were selected for the study.

\section{Operative Procedure}

All patients were operated by single surgeon using
Anteromedial portal technique. Arthroscopy assisted ACL reconstruction with single bundle quadrupled semitendinosus tendon autograft from ipsilateral limb using Endobutton (Smith and Nephew), cortical suspensary fixation method for femoral side and Bioabsorbable interference screw (Smith and Nephew), Aperture fixation method for tibial side.

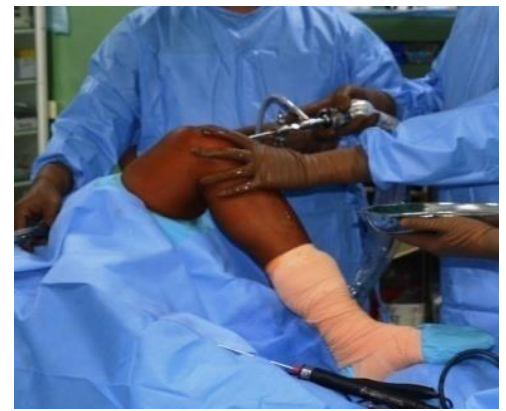

Diagnostic arthroscopy

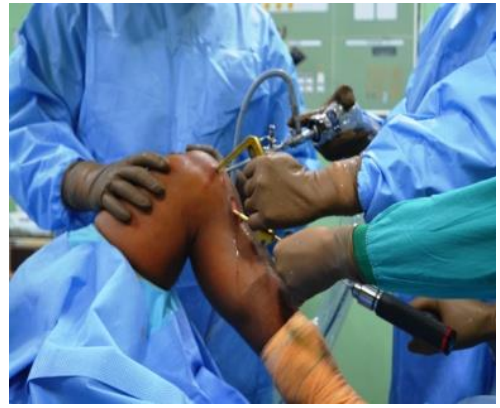

Preparation of tunnel

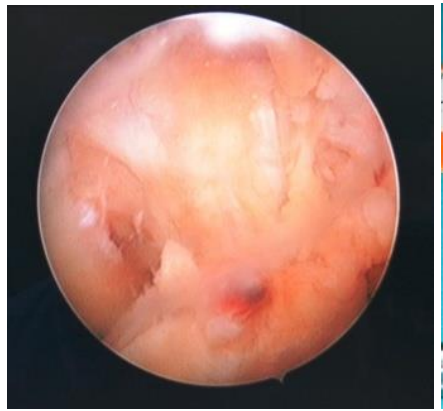

ACL tear

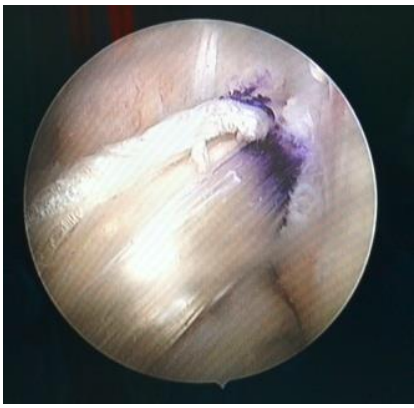

Passage of graft

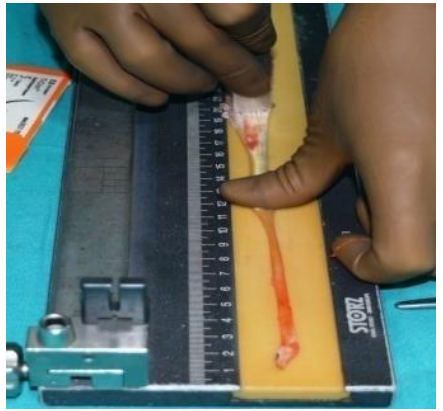

Preparation of ST graft

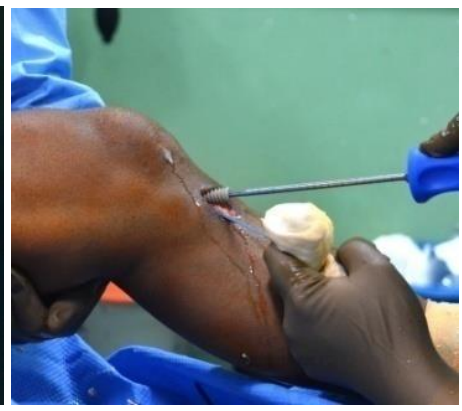

Bioscrew fixation

\section{Post Operative Period}

Immediate post-operative complications like post operative swelling, compartment syndrome, neurological damage and vascular injury is looked for. Patients were hospitalized for 2 weeks post operatively and were sent on 6 weeks medical leave for convalescence.

\section{Follow Up}

The Patients were followed up at regular intervals at 06 weeks, 3months, 6 months, 9 months and at 2 year post operatively. A standard rehabilitation protocol was followed (after Reid, 1992) for all patients. All the patients were subjected to manual laxity testing (Lachman, pivot shift test) and objective laxity is measured by rolimeter. At these intervals all the patients were evaluated by the modified Lysholm knee scoring system (1985). All the patients were also analyzed for their symptoms pre and post operatively.

\section{Results}

\section{Age Distribution}

Table 1

\begin{tabular}{|c|c|c|}
\hline Age in years & Number & Percentage \\
\hline $18-24$ yrs & 41 & $34.2 \%$ \\
\hline $25-31$ yrs & 25 & $20.8 \%$ \\
\hline $32-38$ yrs & 38 & $31.7 \%$ \\
\hline $39-45$ yrs & 16 & $13.3 \%$ \\
\hline Total & 120 & $100 \%$ \\
\hline
\end{tabular}

The analysis of the age groups of the studied cases revealed that the most common affected age group was between 18 to 24 years $(34.2 \%)$ followed by $32-38$ years $(31.7 \%)$ and $25-31$ years $(20.8 \%)$. The mean age of the patient in this study was found to be $30.53 \pm 7.24$ years.

\section{Side of injury}

Table 2

\begin{tabular}{|c|c|c|}
\hline Side of injury & Frequency & Percentage \\
\hline Right & 54 & $45 \%$ \\
\hline Left & 66 & $55 \%$ \\
\hline Total & 120 & $100 \%$ \\
\hline
\end{tabular}

In this study $54(45 \%)$ patients had injury on the right side and $66(55 \%)$ patients had injury on the left side.

\section{Duration from injury to surgery}

Table 3

\begin{tabular}{|c|c|c|}
\hline Duration & Number & Percentage \\
\hline Upto 3 weeks & 0 & $0 \%$ \\
\hline 3 weeks - 3 months & 18 & $15 \%$ \\
\hline 3 months to 6 months & 26 & $21.6 \%$ \\
\hline More than 6 months & 76 & $64.4 \%$ \\
\hline Total & 120 & $100 \%$ \\
\hline
\end{tabular}

In this series no patients were operated within 3 weeks after injury, $18(15 \%)$ patients were operated within 3 weeks-12 weeks (3 months) after injury, 26 (21.6\%) patients were operated within 3 months-6months after injury and 76 $(64.4 \%)$ patients were operated more than 6 months after injury. 
Mode of injury

Table 4

\begin{tabular}{|c|c|c|}
\hline Mode of injury & Number & Percentage \\
\hline Sports/BPET & 79 & $65.8 \%$ \\
\hline RTA & 20 & $16.7 \%$ \\
\hline Fall from height & 15 & $12.5 \%$ \\
\hline Household injuries & 6 & $5 \%$ \\
\hline Total & 120 & $100 \%$ \\
\hline
\end{tabular}

In this series we had $79(65.8 \%)$ patients due to sports/BPET (Battle Physical Efficiency Test) injuries as the most common mode of injury, $26(16.7 \%)$ patients due to RTA, 15(12.5\%) patients due to fall from height and in $6(5 \%)$ patients mode of injury was household injuries.

\section{Presenting symptoms}

Table 5

\begin{tabular}{|c|c|c|}
\hline Symptoms & Frequency & Percentage \\
\hline Pain & 110 & $91.7 \%$ \\
\hline Giving way & 78 & $65 \%$ \\
\hline Recurrent swelling & 47 & $39.2 \%$ \\
\hline Locking & 22 & $18.3 \%$ \\
\hline
\end{tabular}

In this series $110(91.7 \%)$ patients had complaint of pain in the knee at the time of admission, $78(65 \%)$ complaint of giving way sensation of knee, 47(39.2\%) patients complaint of recurrent swelling and 22(18.3\%) complaint of locking sensation of knee.

\section{Graft Size}

Table 6

\begin{tabular}{|c|c|c|}
\hline & Number & Percentage \\
\hline 7 & 18 & $15 \%$ \\
\hline 8 & 64 & $53.3 \%$ \\
\hline 9 & 38 & $31.7 \%$ \\
\hline Total & 120 & $100 \%$ \\
\hline
\end{tabular}

The Graft size used consisted of graft of $7 \mathrm{~mm}, 8 \mathrm{~mm}$ and $9 \mathrm{~mm}$. $8 \mathrm{~mm}$ graft was used in majority $(53.3 \%)$ of the patients followed by $9 \mathrm{~mm}(31.7 \%)$ and $7 \mathrm{~mm}(15 \%)$ grafts. The mean size of graft was $8.2 \pm 0.39$.

\section{Post-operative knee flexion (2 year post op)}

Table 7

\begin{tabular}{|c|c|c|}
\hline Knee flexion & Number & Percentage \\
\hline 0 -120 degrees & 06 & $05 \%$ \\
\hline 0 -130 degrees & 18 & $15 \%$ \\
\hline $0-140$ degrees & 96 & $80 \%$ \\
\hline Total & 120 & $100 \%$ \\
\hline
\end{tabular}

In this study at 2 years follow up, $96(80 \%)$ patients achieved knee flexion from 0-140 degrees, 18 (15\%) patients achieved knee flexion from 0-130 degrees and 6(5\%) patients had knee flexion of less than 130 degrees.

\section{Manual Laxity Tests}

Table 8

\begin{tabular}{|c|c|c|c|c|c|}
\hline \multirow{2}{*}{ Lachman test } & \multicolumn{4}{|c|}{ Lachman test grade } & \multirow{2}{*}{ Total } \\
\cline { 2 - 5 } & Nil & Grade I & Grade II & Grade III & 120 \\
\hline Pre operative & 0 & 12 & 72 & 36 & 120 \\
\hline Post operative (2 year follow up) & 68 & 52 & 0 & 0 & 1 \\
\hline
\end{tabular}

\begin{tabular}{|c|c|c|c|c|c|}
\hline \multirow{2}{*}{ Pivot shift test } & \multicolumn{4}{|c|}{ Pivot shift test grade } & \multirow{2}{*}{ Total } \\
\cline { 2 - 5 } & Nil & Grade I & Grade II & Grade III & 120 \\
\hline Pre operative & 0 & 72 & 44 & 04 & 120 \\
\hline Post operative (2 year follow up) & 112 & 08 & 0 & 0 & 120 \\
\hline
\end{tabular}

There was improvement in the Lachman test at 2 year followup, from grade $1(n=12)$ or grade $2(n=72)$ or grade $3(n=36)$ to grade $1(n=32)$ or grade $0(n=88)$ and There was improvement in the Pivot-shift test at 2 year follow-up, from grade $1(n=72)$ or grade $2(n=44)$ or grade $3(n=04)$ to grade $1(n=08)$ or grade $0(n=112)$.

Objective laxity test (Laxity measurement by Rollimeter):

Table 9

\begin{tabular}{|c|c|c|}
\hline \multirow{2}{*}{ Rollimeter test } & \multicolumn{2}{|c|}{ Rollimeter measurement in mm } \\
\cline { 2 - 3 } & Mean & SD \\
\hline Pre operative & 7.23 & 1.94 \\
\hline Post operative & 3.86 & 1.06 \\
\hline
\end{tabular}

The anterior drawer laxity measurement by rollimeter improved from pre-operative value of $7.23 \pm 1.94$ to post-operative value of $3.86 \pm 1.06$

Lysholm Score

Table 10

\begin{tabular}{|c|c|c|c|c|}
\hline \multirow{2}{*}{ Score (Grading) } & \multicolumn{2}{|c|}{ Pre-operative score } & \multicolumn{2}{c|}{ Post-operative score (2 year post op) } \\
\cline { 2 - 5 } & Number & Percentage & Number & Percentage \\
\hline Less than 65 (Poor) & 48 & $40 \%$ & 0 & $0 \%$ \\
\hline $65-83$ (Fair) & 72 & $60 \%$ & 06 & $05 \%$ \\
\hline $84-90$ (Good) & 0 & $0 \%$ & 60 & $50 \%$ \\
\hline$>90$ (Excellent) & 0 & $0 \%$ & 54 & $45 \%$ \\
\hline Total & 120 & $100 \%$ & 120 & $100 \%$ \\
\hline \multicolumn{4}{|c}{$\sim 50 \sim$}
\end{tabular}


Preoperative and postoperative (after 2 years) Tenger Lysholm scores were compared. While preoperatively $48(40 \%)$ patients had poor Tenger Lysholm scores, 72(60\%) patients had fair Tenger Lysholm scores. Mean preoperative Tegner Lysholm knee score of patients in the present study was $66 \pm 5.2$. Postoperatively 54(45\%) of the patients had excellent, while $60(50 \%)$ had good and only 6(5\%) of patents had fair knee score as per Tegner lysholm scoring system. postoperatively none had poor Tenger Lysholm scores. The mean Tegner Lysholm knee score after 2 years of follow up was $88.7 \pm 5.54$.

Return to pre-injury level of activity (military training activities)

Table 11

\begin{tabular}{|c|c|c|}
\hline Return to pre-injury status & Number & Percentage \\
\hline Yes & 111 & $92.5 \%$ \\
\hline No & 09 & $7.5 \%$ \\
\hline Total & 120 & $100 \%$ \\
\hline
\end{tabular}

After 2 years 111 patients had returned to preinjury level of activity i.e., military training activities

\section{Complications}

Table 12

\begin{tabular}{|c|c|c|}
\hline Complications & Number & Percentage \\
\hline Superficial Infection & 9 & $7.5 \%$ \\
\hline Deep Infection & 0 & $0 \%$ \\
\hline Joint effusion & 6 & $5 \%$ \\
\hline knee pain & 9 & $7.5 \%$ \\
\hline sense of giving away during exertion & 7 & $5.8 \%$ \\
\hline Graft failure & 0 & $0 \%$ \\
\hline Graft site tenderness & 0 & $0 \%$ \\
\hline Thigh muscle wasting (2-3cm) & 19 & $15.8 \%$ \\
\hline
\end{tabular}

In this study, 9 patients had Superficial Infection, 6 patients had Joint effusion in immediate post operative period, 9 patients had knee pain, 7 patients had sense of giving away during exertional activities and 19 patients had Thigh muscle wasting $(2-3 \mathrm{~cm})$

\section{Illustration}

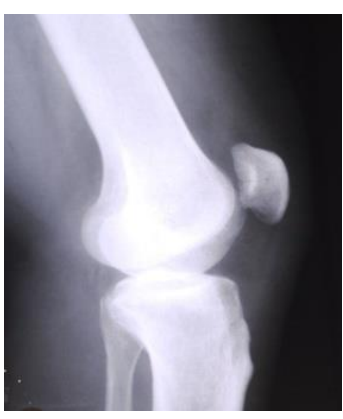

Pre-Operative X-Ray

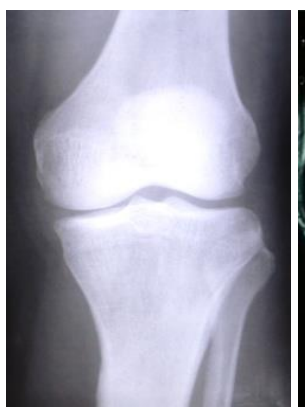

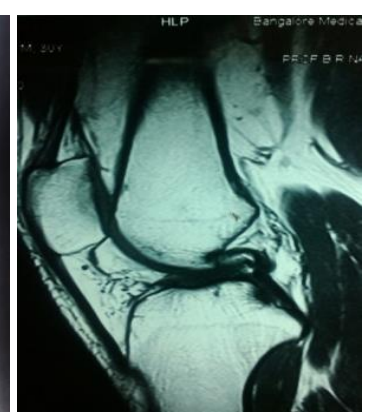

Pre-Operative Mri

\section{Photos (Post Operative)}

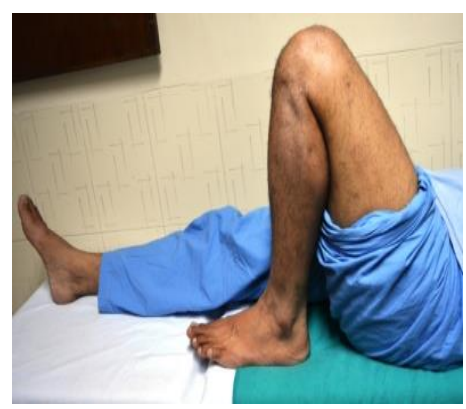

Flexion:

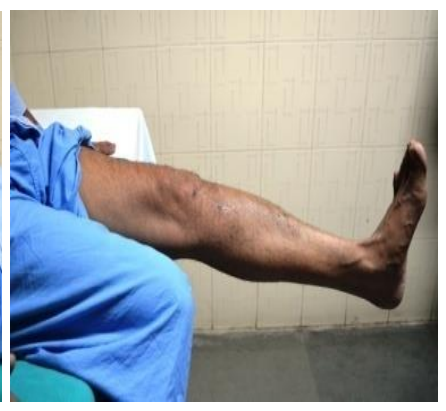

Extension:

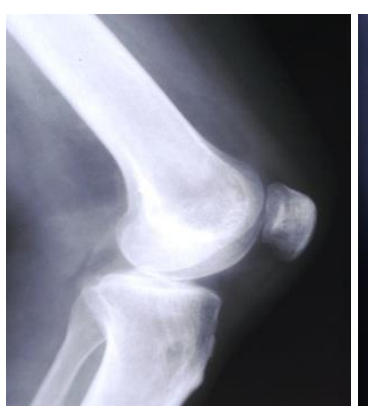

Post-Operative X-Ray:

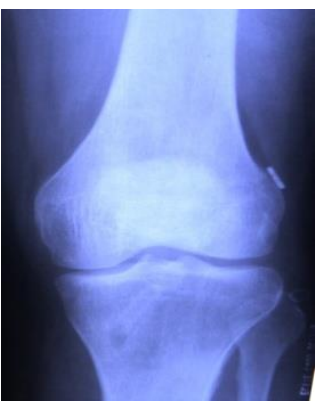

\section{Discussion}

The primary goal of ACL reconstruction is to restore the stability of the knee. Successful clinical outcomes following anterior cruciate ligament reconstruction with a semitendinosus graft has been reported by many authors ${ }^{[19]}$. The choice of fixation in ACL reconstruction is still evolving and the current fixation device which has been widely used were the Endo button and the Bio composite interference screws which has helped to render an improved rehabilitation program post operatively ${ }^{[20]}$.

All patients in our study underwent Arthroscopy assisted ACL reconstruction with single bundle quadrupled semitendinosus tendon autograft from ipsilateral limb using Endobutton (Smith and Nephew), cortical suspensary fixation method for femoral side and Bioabsorbable interference screw (Smith and
Nephew), Aperture fixation method for tibial side.

\section{Age Distribution}

The mean age of patients (in years) in our study was $30.53 \pm 7.24$. The results of our study are comparable to the results of other studies.

Table 13

\begin{tabular}{|c|c|}
\hline Authors & Mean Age \\
\hline Siebold et al. $^{[21]}$ & 29 years \\
\hline Specchiulli et al. ${ }^{[22]}$ & 27 years \\
\hline D Chaudhary et al $^{[23]}$ & 26.8 years \\
\hline N.M. Jomha et al $^{[24]}$ & 26 years \\
\hline D.J. Deehan et al. ${ }^{[25]}$ & 25 years \\
\hline Present study & $30.53 \pm 7.24$ \\
\hline
\end{tabular}




\section{Side of injury}

Brown et al. ${ }^{[26]}$ studied the incidence of the sidedness of limb injury and sex incidence and stated that although their study pointed that females are more prone to this injury, the incidence is yet, more in males due their increased exposure to work in a strenuous environment. They also hypothesed that limb sidedness have no influence in either during injury or the recovery period.

In our study $45 \%$ patients had injury on the right side and $55 \%$ patients had injury on the left side.

Table 14

\begin{tabular}{|c|c|c|}
\hline Authors & Right & Left \\
\hline${\text { D Chaudhary } \text { et al. }{ }^{[23]}}^{[25]}$ & $56.41 \%$ & $43.59 \%$ \\
\hline D.J. Deehan et al. $^{[25]}$ & $61 \%$ & $39 \%$ \\
\hline N.M. Jomha et al. $^{[24]}$ & $45.76 \%$ & $54.24 \%$ \\
\hline Present study $^{2}$ & $45 \%$ & $55 \%$ \\
\hline
\end{tabular}

Mode of Injury

D Chaudhary et al. ${ }^{[23]}$ in their study noted that injury caused by sporting activities accounted for $66.7 \%$ of the patients, whereas motor vehicle accident and household injuries accounted for $30.8 \%$ and $2.5 \%$ respectively. Studies conducted by Patond et al. ${ }^{[27]}$ found sports activities to be the predominant cause of ACL injuries.

All the patients included in the study were serving male soldiers. This was largely attributed to high prevalence of ACL tear among young active soldiers by virtue of their sports and training participation including strenuous physical activities involving jumping, running and obstacle courses. The most common mode of injury $(65.8 \%)$ was sports injury (football, basketball and volleyball game) and during military training (BPET or fall during 9 feet ditch jump, in which soldier has to jump across a 9 feet long ditch with his rifle and backpack). Second common mode (16.7\%) was road traffic accidents.

\section{Duration from Injury to Surgery}

There is no clear consensus on the timing of surgery, although much has been studied so far in the literature. Most authors have opined on waiting for at least 3 weeks before ACL reconstruction from the time of injury ${ }^{[28,29]}$.

In our study we treated acute injuries with bracing and rehabilitation and restricted activity until swelling reduced and the patient regained functional range of motion in the knee. After acute phase subsides, patients with symptoms were then councelled for surgery. Perioperative edema, hyperaemia and ROM appears to influence the outcome of ACL surgery.

\section{Graft Size}

Conte EJ et al. ${ }^{[30]}$ in their systematic review found that ACL reconstruction with quadrupled- strand hamstring autograft with a diameter equal to or larger than $8 \mathrm{~mm}$ decreases failure rates. Mariscalco MW et al. [31] in their multicentric retrospective chart review of prospectively collected cohort data of 263 patients undergoing primary ACL reconstruction with hamstring autograft, found that smaller hamstring autograft size ( $8 \mathrm{~mm}$ or smaller) is a predictor of poorer KOOS sport/recreation function 2 years after primary ACL reconstruction. Treme et al. ${ }^{[32]}$ proposed that graft length is related to the height and BMI of the patient, while the diameter is related to the thickness of the thigh. He further opined that a graft diameter of $<7 \mathrm{~mm}$ will have a higher risk of failure.
In our study, $8 \mathrm{~mm}$ graft was used in majority (53.3\%) of patients followed by $9 \mathrm{~mm}(31.7 \%)$ and $7 \mathrm{~mm}(15 \%)$ grafts. The mean size of graft was $8.2 \pm 0.39$.

\section{Laxity Tests}

Manual laxity testing of all the patient was carried out by Anterior Drawer test, Lachman test and Pivot shift test. None of the patients had grade III laxity 02 year post-operatively. Post-operatively at 02 year follow up, 08 case $(6.7 \%)$ had a positive Pivot shift test with a "Glide" (Grade-I), whereas remaining 112 cases $(93.3 \%)$ had a negative Pivot shift test. The mean pre-operative rolimeter measurement was $7.23 \pm 1.94$ SD. The mean postoperative rolimeter measurement at 02 year follow up was $3.86 \pm 1.06 \mathrm{SD}$. The results of pre and postoperative measurement by rolimeter were statistically analyzed and found statistically significant. Thus laxity tests performed at 02 year follow-up was statistically significant suggesting improvement in the stability of knee between pre-operative and post-operative status of patients in our study.

These results are comparable to the results achieved by various other authors Beynnon et al. ${ }^{[17]}$ Saccomanno et al. ${ }^{[33]}$ and Poolman et al. ${ }^{[34]}$

\section{Functional Outcome}

H. E. Bourke et al. [35] reported the outcome of 'isolated' anterior cruciate ligament (ACL) ruptures treated with anatomical endoscopic reconstruction using hamstring tendon autograft at a mean of 15 years. A total of 152 patients underwent subjective assessment at 15 years. The mean Lysholm knee score at 15 years was 93 .

Daniel B. O`Neill et al. ${ }^{\text {[37] }}$ evaluated 125 patients with anterior cruciate ligament rupture after a mean duration of 42 months. 40 patients(Group 1) were treated with two incision reconstruction using autogenous semitendinosus gracilis autograft, 40 patients(Group 2) were treated with two incision reconstruction using autogenous bone patellar tendon bone graft and 45 patients (Group 3) were treated with single incision (endoscopic technique) reconstruction using autogenous bone patellar tendon bone graft. Lysholm score was $>90$ in $88 \%$ in group $1,90 \%$ in group 2 and $93 \%$ in group 3.

Williams et al. ${ }^{[38]}$ in their study, reported outcome that the mean score improved from 55 points to 91 points post operatively at a 2 year follow up $(\mathrm{p}<0.01)$.

Fareed et al. ${ }^{[39]}$ reported the results of a retrospective study on patients who underwent arthroscopic ACL reconstruction. The average follow up was 25.4 months. A satisfactory outcome was seen in $96 \%$. Cooley et al. ${ }^{[40]}$ in their study concluded that ACL reconstruction using quadrupled fold semitendinosus tendon autograft About $85 \%$ of the patients according to Lysholm scoring fall into normal to near normal in their study. Gulick et al. ${ }^{[42]}$ in 2002 studied on 57 patients and concluded that $84 \%$ of their patients returned to pre injury level of function.

In our study, all patients strictly followed the rehabilitation protocol. Patients were not allowed to return to full active military duty or sports activities during their rehabilitation program and they were placed in low medical category mentioning their restricted employability. Lysholm score was recorded at 6 months, 1 year and 02 year postoperatively. At 02 year follow up post-operatively, the mean postoperative Lysholm score improved from pre-operative score of $66 \pm 5.2$ to $88.70 \pm 5.54$. The improvement in the mean pre-operatve and post-operative Lysholm scores was statistical significant 
$(\mathrm{p}<0.01)$. At 02 year follow up, 54 cases $(45 \%)$ had excellent score (>90). Good score (84-90) was seen in 60 cases $(50 \%)$. The overall satisfactory result (excellent + good) at 02 year follow up was $95 \%$. Out of this, 111 patients $(92.5 \%)$ were able to achieve preinjury activity level. They were upgraded and considered fit to resume all active military duties.

\section{Complications}

Judd et al. ${ }^{[41]}$ reported that hamstring grafts were associated with a higher incidence of infection. Soft tissue injuries involved in harvesting the tendons and creating a tibial tunnel and the use of an additional metallic post, washer, and braided sutures in subcutaneous position could be the causes of wound complications. With proper wound closure and burying of hardware under soft tissue sleeve may minimize this complication. Kim HJ et al. ${ }^{[42]}$ in their short study reported 7 cases $(7.1 \%)$ of infection ( 5 deep and 2 superficial) in 98 cases of anterior cruciate ligament (ACL) reconstruction using hamstring autografts in a 17-month period.

Leo Chan et al. ${ }^{[43]}$ Reported that the technique of ACL reconstruction using quadrupled fold semitendinosus tendon autograft for ACL reconstruction using the Endobutton for femoral fixation has been used for over ten years with no known instance of fixation failure.

Ian et al. ${ }^{[44]}$ and Aglietti et al. ${ }^{[45]}$ in their respective studies concluded that in ACL reconstruction using hamsting graft, the postoperative ROM following rehabilitation protocol was almost equal to the pre injury status at the end of follow up.

D. J. Deeham et al. ${ }^{[25]}$ in their study noted that $87 \%$ had wasting of less than $1 \mathrm{~cm}, 9 \%$ had a wasting of $1-2 \mathrm{cms}$ and $4 \%$ had wasting of $3 \mathrm{cms}$. N.M. Jomha ${ }^{[26]}$ et al. in their study noted that $66.1 \%$ patients had no thigh atrophy, $27.12 \%$ patients had thigh atrophy of less than $1 \mathrm{~cm}$ and $6.78 \%$ patients had thigh atrophy of 1-2 cms.

In our study, Postoperatively total of 9 cases developed superficial wound infection. All of them settled with proper wound care and antibiotics. 6 case $(5 \%)$ had swelling in the knee joint post op period. The patients were monitored regularly and settled gradually over 6 weeks. At 2 year follow up, 9 cases $(7.5 \%)$ complained of knee pain, 7 cases $(5.8 \%)$ complained of a slight sense of giving away during exertional activity or sports activity. Thigh muscle wasting of $2-3 \mathrm{cms}$ measured $15 \mathrm{cms}$ above the superior pole of patella or at maximum girth site were found in 19 out of 120 patients i.e. $15.8 \%$. All patients in our study had postoperative ROM of $>120$ degress. There was no case of deep infection, graft failure or graft side tenderness noted.

\section{Conclusion}

This study included 120 patients (serving soldiers) suffering from ACL deficiency in the age group of $18-45$ years, with minimum of 2 years follow up. The aim of study was to evaluate functional outcome of arthroscopy assisted ACL reconstruction with quadrupled hamstring tendon graft using Endobutton and Bioabsorbable interference screw.

From this study, we conclude that anterior cruciate ligament injuries are common in younger age group individuals. Sports related injury and RTA are the most common modes of injury. Arthroscopy assisted ACL reconstruction using Semitendinosus tendon autograft provides a stable knee, reduces postoperative morbidity and enables early rehabilitation. Graft fixation using endobutton and and Bioabsorbable interference screw will help the graft to facilitate graft tunnel healing and also maintain its strength until there is a good graft to bone healing occurs. The functional outcome is excellent to good and allows the patients to return to preinjury level of activity, allows the patient to squat, sit crossed leg and climb stairs without difficulty. Arthroscopy assisted ACL reconstruction with quadrupled Semitendinosus tendon autograft using Endobutton and Bioabsorbable interference screw is an excellent treatment option for anterior cruciate ligament deficient knees. Degeneration of the articular surfaces rarely occurs and the re-operation rates are minimal.

Management of anterior cruciate ligament tear requires careful pre-operative planning, patient selection, radiological evaluation graft with appropriate thickness, Timing of surgery, meticulous intraoperative care, precision in technique and post-operative rehabilitation including detailed counselling for good functional outcome.

\section{Declarations \\ Funding: None}

Conflict of interest: None declared

\section{References}

1. Cor van der Hart P, Michel PJ, van den Bekerom, Thomas Patt W. The occurrence of osteoarthritis at a minimum of ten years after reconstruction of the anterior cruciate ligament; Journal of Orthopaedic Surgery and Research. 2008; 3:24.

2. Matjaz Sajovic. Simultaneous Bilateral Anterior Cruciate Ligament (ACL) Reconstruction with use of Hamstring Tendon Autografts: a Case Report. Journal of Surgery. 2013; 1(1):1-5.

3. Adil Ajuied, Christian Smith, Fabian Wong, Simon Hoskinson, Diane Back, Andrew Davies. A Survey of Rehabilitation Regimens Following Isolated ACL Reconstruction. JMED Research, 2014, 1-9.

4. Kostas Patras a, Giorgos Ziogas a, Stavros Ristanis a, Elias Tsepis ab, Nicholas Stergiou ac, Anastasios Georgoulis Da. ACL reconstructed patients with a BPTB graft present an impaired vastus lateralis neuromuscular response during high intensity running. Journal of Science and Medicine in Sport. 2010; 13:573-577.

5. Anthony M, Buoncristiani MD, Fotios P, Tjoumakaris MD, James Starman S, Mario Ferretti BS et al. Anatomic Double-Bundle Anterior Cruciate Ligament Reconstruction. Arthroscopy: The Journal of Arthroscopic and Related Surgery. 2006; 22(9):10001006.

6. Terry Canale S, James Beaty H. Arthroscopy of the Lower Extremity: Campbell's operative orthopaedics, $11^{\text {th }}$ edition, 2855-2856.

7. Ryan Lobb, Steve Tumilty, Leica Claydon S. A review of systematic reviews on anterior cruciate ligament reconstruction rehabilitation. Physical Therapy in Sport 2012; xxx:1-9.

8. Yau WP, Fok AWM, Yee DKH. Tunnel Positions in Transportal Versus Transtibial Anterior Cruciate Ligament Reconstruction: A Case-Control Magnetic Resonance Imaging Study. Arthroscopy: The Journal of Arthroscopic \& Related Surgery. 2013; 29(6):1047-52. DOI: https://doi.org/10.1016/j.arthro.2013.02.010

9. Cirstoiu C, Circota G, Panaitescu C, Niculaita R. The Advantage of Arthroscopic Anterior Cruciate Ligament Reconstruction with Autograft from the Tendons of the Semitendinosus - Gracilis Muscles for the Recovery of the Stability of the Knee. Mædica. 2011; 6(2):109-13. 
10. Friedman MJ. Arthroscopic semitendinosus (gracilis) reconstruction for anterior cruciate ligament deficiency. Tech Orthop. 1988; 2:74-80.

11. Streich NA, Reichenbacher S, Barié A, Buchner M, Schmitt H. Long-term outcome of anterior cruciate ligament reconstruction with an autologous four-strand semitendinosus tendon autograft. International Orthopaedics. 2013; 37(2):279-284.

12. Milano G, Mulas PD, Ziranu F, Piras S, Manunta A, Fabbriciani C. Comparison between different femoral fixation devices for ACL reconstruction with doubled hamstring ten $\neg$ don graft: a biomechanical analysis. Arthroscopy. 2006; 22:660-8.

13. Petre BM, Smith SD, Jansson KS, de Meijer PP, Hackett TR, LaPrade RF et al. Femoral cortical suspension devices for soft tissue anterior cruciate ligament reconstruction: a comparative study. Am J Sports Med. 2013; 41(1):416-22. $\quad$ PMID: 23263298 DOI: $10.1177 / 0363546512469875$

14. Johnson JS, Smith SD, LaPadre CM, Turnbull TL, LaPadre RF, Wijdicks CA. A biomechanical comparision of femoral cortical suspension devices for soft tissue anterior cruciate ligament reconstruction ubder high loads. Am J Sports Med. 2015; 43(1):154-60. PMID:25326014 DOI:10.1177/0363546514553779

15. More ADO, Pizzolatti ALA, Fancello EA, Roesler CRdM. ri Digiacomo Ocampo et al. Biomechanical performances of Bio Cross Pin and Endobutton for ACL reconstruction at femoral side: a porcine model. Res. Biomed Eng[online]. 2016; 36:28-34. DOI: http://dx.doi.org/10.1590/2446-4740.0720

16. Zeng C, Lei G, Gao S, Luo W. Methods and devices for graft fixation in anterior cruciate ligament reconstruction (Protocol). Cochrane Database of Systematic Reviews, 2013, 9. Art. No.: CD010730. DOI: 10.1002/14651858.CD010730.

17. Beynnon B, Johnson R, Fleming B, Kannus P, Kaplan P, Samani J. Anterior cruciate ligament replacement: comparison of bone-patellar tendonbone grafts with twostrand hamstring grafts. J Bone Joint Surg Am. 2002; 84(9):1503-13.

18. Ejerhed L, Kartus J, Sernert N, Kohler K, Karlsson J. Patellar tendon or semitendinosus tendon autografts for anterior cruciate ligament reconstruction? A prospective randomized study with a two-year follow-up. Am J Sports Med. 2003; 31(1):19-25.

19. Aglietti P, Buzzi R, Menchetti PM, Giron F. Arthroscopically assisted semitendinosus and gracilis tendon graft in reconstruction for acute anterior cruciate ligament injuries in athletes. Am J Sports Med. 1996; 24:726-31.

20. Freedman KB, D'Amato MJ, Nedeff DD, Kaz A, Bach BR Jr. Arthroscopic anterior cruciate ligament reconstruction. A meta-analysis comparing patellar tendon and hamstring tendon autografts. Am J Sports Med. 2003; 31(1):2-11.

21. Specchiuli F, Laforgia Mocci, Miotta Scialpi, Solaring. Anterior cruciate ligament reconstruction: A comparison of 2 techniques. Clin Orthop. 1995; 311:142-7.

22. Siebold R, Dehler C, Ellert T. Prospective randomized comparison of double-bundle versus single bundle anterior cruciate ligament reconstruction. J Arth Related Surg. 2008; 24:137-45.

23. Chaudhary D, Monga P, Joshi D, Easwaran R, Bhatia N, Singh AK. Arthroscopic reconstruction of the anterior cruciate ligament using bone-patellar tendonbone autograft: Experience of the first 100 cases. Journal of Orthopaedic Surgery. 2005; 13(2):147-152.

24. Jomha NM, Pinczewski LA, Clingeleffer A, Otto DD. Arthroscopic reconstruction of the anterior cruciate ligament with patellar-tendon autograft and interference screw fixation; J Bone Joint Surg.(Br). 1999; 81-B:775-9.

25. Deehan DJ, Salmon LJ, Webb VJ, Davies ALA. Pinczewski-Endoscopic reconstruction of the anterior cruciate ligament with an ipsilateral patellar tendon autograft, J Bone Joint Surg. (Br). 2000; 82-B; 984-91.

26. Brown TN, Palmeri-Smith RM, Mc Lean SG. Sex and limb differences in hip and knee kinematics and kinetics during anticipated and unanticipated jump landings: implications for anterior cruciate ligament injury. British J Sports Med. 2009; 43:1049-56.

27. Patond KR, Chauhan VD, Kumar N. Semitendinosus transfer for anterior cruciate ligament insufficiency. Ind J of orthopaedics. 1992; 26:1.

28. Shelbourne KD, Wilcken JH, Mollabashy A, DeCarlo M. Arthrofibrosis in acute anterior cruciate ligament reconstruction. The effect of timing of reconstruction and rehabilitation. Am J Sports Med. 1991; 19(4):332-6.

29. Shelbourne KD, Patel DV. Timing of surgery in anterior cruciate ligament-injured knees. Knee Surg Sports Traumatol Arthrosc. 1995; 3(3):148-56.

30. Conte EJ, Hyatt AE, Gatt CJ, Dhawan A. Hamstring Autograft Size Can Be Predicted and Is a Potential Risk Factor for Anterior Cruciate Ligament Reconstruction Failure. Arthoscopy. 2014; 3(7):882-90. DOI: http://dx.doi.org/10.1016/j.arthro.2014.03.028

31. Marscalo MW, Flanigan DC, Mitchell J, Pedroza AD, Jones MH, Andrish JT et al. The Influence of Hamstring Autograft Size on Patient Reported Outcomes and Risk of Revision After Anterior Cruciate Ligament Reconstruction: A Multicenter Orthopaedic Outcomes Network (MOON) Cohort Study. Arthroscopy: The Journal of Arthroscopic and Related Surgery. 2013; 29(12):1948-53. http://dx.doi.org/10.1016/j.arthro.2013.08.025

32. Treme G, Diduch DR, Billante MJ, Miller MD, Hart JM. Hamstring graft size prediction: a prospective clinical evaluation. Am J Sports Med. 2008; 36(11):2004-9.

33. Saccomanno MF, Shin JJ, Mascarenhas R, Haro M, Verma NN, Cole BJ et al. Clinical and functional outcomes after anterior cruciate ligament reconstruction using cortical button fixation versus transfemoral suspensory fixation: a systematic review of randomized controlled trials. Arthroscopy. 2014; 30(11):1491-8.

34. Poolman RW, Farrokhyar F, Bhandari M. Hamstring tendon autograft better than bone patellar-tendon bone autograft in ACL reconstruction: A cumulative metaanalysis and clinically relevant sensitivity analysis applied to a previously published analysis. Acta Orthop. 2007; 78:350-4.

35. Bourke HE, Gordon DJ, Salmon LJ, Waller A, Linklater $\mathrm{J}$, Pinczewski LA. The outcome at 15 years of endoscopic acl reconstruction using hamstring tendon autograft. J Bone Joint Surg Br. 2012; 94-B:630-7.

36. Daniel B, O’Neill MD. Nassau Bay-Arthroscopic Assisted Reconstruction of the Anterior Cruciate Ligament. The journal of Bone and Joint Surgery. 1996; 78A(6):803-813.

37. Williams III RJ, Hyman J, Petrigalino F, Rozental T, Wickiewicz TL. Anterior ligament reconstruction with a 
four strand quadruple hamstring tendon autograft. J Bone Joint Surg. 2004; 86:225-32.

38. Fareed H, Dionellis P, Paterson FW. Arthroscopic ACL Reconstruction using 4 strand hamstring tendon graft. J Bone Join Surg. 2003; 85:231-6.

39. Cooley V, Deffner K, Rosenberg T. Quadrupled semitendinosus anterior cruciate ligament reconstruction: 5 -year results in patients without meniscus loss. Arthroscopy. 2001; 17:795-800.

40. Gulick TD, Yoder HN. Anterior cruciate ligament reconstruction: Clinical outcomes of patella tendon and hamstring tendon grafts. J Sports Sci Med. 2002; 1:63-71.

41. Judd D, Bottoni C, Kim D, Burke M, Hooker S. Infections following arthroscopic anterior cruciate ligament reconstruction. Arthroscopy. 2006; 22:375-84.

42. Kim HJ, Lee HJ, Lee JC, Min SG, Kyung HS. Evaluation of Infection after Anterior Cruciate Ligament Reconstruction during a Short Period. Knee Surg Relat Res. 2017; 29(1):45-51.

43. Chen L, Cooley V, Rosenberg T. ACL reconstruction with hamstring tendon. Orthop Clin North Am. 2003; 34(1):9-18.

44. Corry IS, Webb JM, Clingeleffer AJ, Pinczewski LA. Arthroscopic Reconstruction of the Anterior Cruciate Ligament A Comparison of Patellar Tendon Autograft and Four-Strand Hamstring Tendon Autograft. Am J Sports Med. 1999; 27(4):444-54.

45. Aglietti P, Buzzi R, Zaccherotti G, De Biase P. Patellar tendon versus doubled semitendinosus and gracilis tendons for anterior cruciate ligament reconstruction. Am J Sports Med. 1994; 22:211-8. 